\title{
Technikaffinität als Ressource für die Arbeit in Industrie 4.0
}

\author{
Christiane Attig ${ }^{1}$, Sebastian Mach ${ }^{2}$, Daniel Wessel ${ }^{3}$, Thomas Franke ${ }^{4}$, \\ Franziska Schmalfuß ${ }^{5}$, Josef F. Krems ${ }^{6}$ \\ ${ }^{1}$ christiane.attig@psychologie.tu-chemnitz.de \\ ${ }^{2}$ sebastian.mach@psychologie.tu-chemnitz.de \\ ${ }^{3}$ wessel@imis.uni-luebeck.de \\ ${ }^{4}$ franke@imis.uni-luebeck.de \\ ${ }^{5}$ franziska.schmalfuss@psychologie.tu-chemnitz.de \\ ${ }^{6}$ krems@psychologie.tu-chemnitz.de \\ 1,2,5,6 Institut für Psychologie, Allgemeine und Arbeitspsychologie, \\ Technische Universität Chemnitz, Deutschland \\ ${ }^{3,4}$ Institut für Multimediale und Interaktive Systeme (IMIS), Ingenieurpsychologie und \\ Kognitive Ergonomie, Universität zu Lübeck, Deutschland
}

DOI: 10.14464/awic.v3i0.251

\section{ABSTRACT}

Die zunehmende Digitalisierung der Arbeitswelt bietet nicht nur vielversprechende Innovationen zur arbeiterzentrierten, adaptiven Aufgabengestaltung, sondern stellt den Einzelnen auch vor die Herausforderung, in höherem Maße mit digitaler Technik zu interagieren. Für die erfolgreiche Interaktion mit Technik sind zwei Faktoren ausschlaggebend: Wissen über bzw. Erfahrung mit technischen Systemen sowie Persönlichkeitsaspekte, die sich als Interaktionsstil im Umgang mit Technik manifestieren. Wir präsentieren das Konzept der interaktionsbezogenen Technikaffinität (affinity for technology interaction, ATI) als zentrale Ressource zum erfolgreichen Umgang mit Technik, und mit der ATI-Skala eine ökonomische und reliable Skala zur Quantifizierung der ATI eines Nutzers. Neben bestehenden Evaluationsergebnissen $(N>1500)$ stellen wir erste Ergebnisse einer Nutzerstudie vor, die im Rahmen des Projektes Factory2Fit durchgeführt wurde. Die Probanden interagierten mit 3DDruckern und erlebten unterschiedliche Automatisierungsgrade des Prozesses. Zusätzlich zum Automatisierungsgrad wurde die Erfahrung der Probanden durch unterschiedliche Trainingsintensitäten variiert. Die Ergebnisse liefern erste Hinweise auf die Relevanz von ATI für die Vorhersage des Interaktionserfolgs mit Technologie und damit für UsabilityEvaluationen in Industrie 4.0-Umgebungen. 
Keywords: Technikaffinität, Mensch-Technik-Interaktion, Industrie 4.0

\section{EINLEITUNG}

Unsere Arbeitswelt ist in zunehmendem Maße durch Vernetzung von Mensch und Maschine, Automation und Assistenz charakterisiert (Hermann, Pentek, \& Otto, 2016). Daraus erwächst die Anforderung an die Arbeitenden stärker mit digitaler Technik zu interagieren, beispielsweise in den Bereichen der Prozessüberwachung und -kontrolle, Nutzung von Robotersystemen oder der Anwendung von Virtual und Augmented Reality zu Assistenz- oder Trainingszwecken (Pinzone et al. 2017). Die erfolgreiche Interaktion mit digitalen Geräten ist somit eine wichtige Voraussetzung für hohe Arbeitsleistung (Windlinger, Nenonen \& Airo, 2015), aber auch ausschlaggebend für die vom Arbeiter wahrgenommene Usability und die allgemeine User Experience (Mahmood, Burn, Gemoets, \& Jacquez, 2000).

Neben persönlichen Ressourcen wie Wissen über bzw. Erfahrung mit technischen Geräten spielen auch Persönlichkeitseigenschaften für die erfolgreiche Technikinteraktion eine wichtige Rolle (Attig, Wessel, \& Franke, 2017; Pocius, 1991). Insbesondere Persönlichkeitsaspekte, die sich als Interaktionsstil (d.h. Annäherung vs. Vermeidung) im Umgang mit Technik manifestieren, können als wesentlich für die Technikinteraktion angenommen werden (Franke, Attig, \& Wessel, 2018), speziell die interaktionsbezogene Technikaffinität (affinity for technology interaction, ATI). Sie ist definiert als die interindividuell variierende Tendenz, sich intensiv mit Technik auseinanderzusetzen (Franke et al., 2018). Die Charakterisierung der interaktionsbezogenen Technikaffinität kann nicht nur Aufschluss darüber geben, wie hoch die Passung zwischen Individuum und Arbeitsanforderung hinsichtlich der Interaktion mit digitaler Technik ist. Auch für die Forschung und Entwicklung im Kontext von Industrie 4.0 ist es zentral, die ATI-Werte der Probanden bei UsabilityEvaluationen zu kennen. Erste Ergebnisse legen nahe, dass eine höhere interaktionsbezogene Technikaffinität mit einer besser eingeschätzten Usability einhergeht (Attig, Wessel \& Franke, 2018). Für die Beurteilung der Gebrauchstauglichkeit neuer digitaler Technik muss die Stichprobe also die ATI-Werte der späteren Nutzer widerspiegeln.

Zur Charakterisierung der interaktionsbezogenen Technikaffinität wurde mit der ATI-Skala ein ökonomisches, reliables und valides Instrument vorgelegt (siehe Abschnitt 1.1). Noch nicht abschließend geklärt ist jedoch die Frage, inwieweit die ATI-Skala zur Vorhersage des Erfolgs beim Umgang mit Technik geeignet ist (d.h. Daten zur Kriteriumsvalidität stehen noch aus). Ziel der vorliegenden Arbeit bildet daher die Prüfung der Validität der ATI-Skala zur Vorhersage des Interaktionserfolgs mit Technologie in Industrie 4.0-Umgebungen.

\subsection{DIE ATI-SKALA: ENTWICKLUNG, RELIABILITÄT UND VALIDITÄT}

Die ATI-Skala wurde entsprechend ihrer theoretischen Konzeptualisierung als domänenspezifische Manifestation des need for cognition (NFC, die intrinsischen Motivation für intensive kognitive Beschäftigung mit Sachverhalten; Cacioppo \& Petty, 1982), in 
Anlehnung an die deutsche NFC-Kurzskala (Bless, Wänke, Bohner, Fellhauer, \& Schwarz, 1994) entwickelt. Darüber hinaus wurden Items generiert, die die Tendenz zur intensiven Technikinteraktion abbilden. Die Skala enthält insgesamt neun Items, die auf einer 6-stufigen Likert-Skala von stimmt gar nicht bis stimmt völlig beantwortet werden (für die Online-Version und weitere Informationen siehe www.ati-scale.org). Die durchschnittliche Bearbeitungszeit liegt bei unter zwei Minuten.

Zur Erfassung der Dimensionalität, Reliabilität und Konstruktvalidität wurden bisher fünf Stichproben mit insgesamt $N=1576$ Teilnehmern erhoben (für die komplette Methodik und Ergebnisdarstellung siehe Franke et al., 2018). Explorative Faktorenanalysen ergaben Einfaktorenlösungen, sodass von Unidimensionalität ausgegangen werden kann. Als Indikator für die Skalenreliabilität wurde die interne Konsistenz (Cronbachs $\alpha$ ) herangezogen, welche in den Stichproben zwischen $\alpha=.83$ und $\alpha=.92$ liegt und somit als gut bis exzellent interpretiert werden kann (vgl. Cripps, 2017). Zur Beurteilung der Konstruktvalidität wurden Korrelationen mit konstruktnahen sowie konstruktfernen Fragebögen herangezogen. Diese zeigten, dass die ATI erwartungsgemäß moderat bis stark mit den Konstrukten NFC, Geekism (Schmettow \& Drees, 2014), Technikbegeisterung (Karrer, Glaser, Clemens, \& Bruder, 2009), Computerangst (Richter, Naumann \& Horz, 2010) und Kontrollüberzeugungen im Umgang mit Technik (Beier, 1999) korreliert. Auch die Korrelationen zu selbstberichtetem technischen Problemlöseerfolg und technischen Systemlernerfolg waren erwartungsgemäß hoch. Mit den als konstruktfern angenommenen Persönlichkeitsdimensionen der Big Five zeigten sich, ebenfalls erwartungsgemäß, keine bis schwache Korrelationen. Zusammenfassend kann die ATI-Skala als ökonomisches, reliables und konstruktvalides Instrument bezeichnet werden (Franke et al., 2018).

\subsection{FRAGESTELLUNG UND HYPOTHESEN}

Zur Erfassung der Kriteriumsvalidität werden Zusammenhänge zwischen dem Testwert und relevanten Außenkriterien untersucht (Moosbrugger \& Kelava, 2012). Da ATI definiert ist als interindividuell variierende Tendenz sich intensiv mit Technik auseinanderzusetzen, welche in der erfolgreichen Technikinteraktion resultieren sollte, sind Indikatoren für ebendiesen Erfolg als mögliche Außenkriterien geeignet. In der vorgestellten Studie untersuchen wir daher Maße für die objektive Performanz sowie für den subjektiven Workload als prototypische Indikatoren des Technikinteraktionserfolgs mit 3D-Druckern in einer Industrieumgebung. Diese sollen Hinweise zur Beantwortung der Forschungsfrage (Q1) geben: Wie hängt die ATI-Skala mit dem Technikinteraktionserfolg in Arbeitsumgebungen zusammen? Wir nehmen an, dass $(\mathrm{H} 1)$ der ATI-Score negativ mit der Interaktionszeit korreliert. Weiterhin nehmen wir an, dass $(\mathrm{H} 2)$ der ATI-Score negativ mit dem subjektiven Workload der Probanden korreliert. Essentiell ist darüber hinaus die Frage, ob der ATI-Score oder das Durchlaufen eines angeleiteten Trainings stärker mit der Interaktionszeit bzw. dem subjektiven Workload zusammenhängt. Diese Forschungsfrage (Q2) wird explorativ untersucht. 


\section{METHODE}

\subsection{STICHPROBE}

An der Studie, welche im April bis Mai 2018 in der Experimental- und Digitalfabrik (EDF) der TU Chemnitz (https://www.tu-chemnitz.de/mb/FabrPlan/edf.php) in Zusammenarbeit mit der Professur für Fabrikplanung und Fabrikbetrieb stattfand, nahmen bis zum gegenwärtigen Zeitpunkt $N=16$ Personen teil. Die Rekrutierung erfolgte über studentische Mailverteiler der TU Chemnitz. Das durchschnittliche Alter der Probanden lag bei $M=23,6(S D=4,3)$ und fünf von innen (31\%) waren weiblich. Alle Probanden studierten Psychologie (44\%) oder Sensorik und Kognitive Psychologie (56\%).

\subsection{ABLAUF, MATERIAL UND GERÄTE}

Nach ihrer Ankunft in der EDF und der Begrüßung durch den Versuchsleiter füllten die Probanden eine Einwilligungserklärung sowie eine Datenschutzerklärung aus. Nachdem sie sich entsprechend ihrer Händigkeit eine Smartwatch (Samsung Gear S3) an ihren nicht dominanten Arm anlegten, füllten sie einen demographischen Fragebogen sowie die ATISkala (Franke et al., 2018) aus.

Im Anschluss interagierten sie mit zwei 3D-Druckern der Firma Ultimaker 2 Go. Zur Variation des Trainings wurden die Probanden in zwei Gruppen aufgeteilt: Eine Gruppe erhielt keine Einführung in die Benutzung des Druckers und konnte lediglich eine schriftliche Anleitung benutzen (kein Training), die zweite Gruppe erhielt eine intensive Anleitung durch den Versuchsleiter sowie eine schriftliche Anleitung und führte gemeinsam mit dem Versuchsleiter im Vorhinein die Versuchsbedingungen teilweise durch (angeleitetes Training). Die eigentliche Aufgabe bestand anschließend darin, drei unterschiedliche Druckerzeugnisse (Würfel, Aufwickler für Kopfhörer, Klemme) zu produzieren und zu vermessen, wobei zusätzlich der Automatisierungsgrad der Produktion (manuell, halbautomatisch, automatisch) variiert wurde. Die Aufgabenstellung (d.h. welches Teil mit welchem Automatisierungslevel auf welchem Drucker erzeugt werden soll) wurde randomisiert und auf einer Beamerwand sowie auf der Smartwatch dargestellt. Nach jedem produzierten Druckerzeugnis füllten die Probanden zur Erfassung ihres Workloads den NASA-TLX (Hart \& Staveland, 1988) aus.

Während des gesamten Versuchsablaufs wurden die Probanden mit zwei verschiedenen Kameras erfasst. Die Analyse der Interaktionszeit basierte auf der Annotation der Videos mittels ELAN Version 5.2 (Max-Planck-Institut für Psycholinguistik, 2018). Die Interaktionszeit umfasst die gesamte Bearbeitungszeit ohne Wartezeiten sowie sonstige Tätigkeiten, die nicht Teil der Aufgabendurchführung waren. Als Beginn der Bearbeitungszeit wurde der Zeitpunkt festgelegt, zu dem die Aufgabe über den Bildschirm und auf der Smartwatch angezeigt wurde. Als Ende wurde der Zeitpunkt festgelegt, als die Probanden den Wert der Messung des fertiggestellten Produktes in das Messprotokoll eingetragen hatten. Die Probanden wurden dazu angehalten, die angezeigten Aufgaben möglichst zügig zu absolvieren. Aus technischen 
Gründen war die Videoannotation für zwei Probanden nicht möglich, daher sank die Stichprobengröße bzgl. der Interaktionszeit auf $n=14$.

Für die Analyse der Zusammenhänge zwischen ATI und Interaktionszeit sowie subjektivem Workload wurde jeweils ein Mittelwert über alle Bedingungen hinweg gebildet. Die Betrachtung von Unterschieden in Performanz und Workload hinsichtlich des Automatisierungsgrad sowie die Interaktion mit der Smartwatch sind nicht Gegenstand der vorliegenden Arbeit.

\section{ERGEBNISSE}

Da die Datensammlung noch nicht abgeschlossen ist, sind die präsentierten Ergebnisse als vorläufig zu betrachten. Deskriptive Statistiken der untersuchten Variablen ATI, Interaktionszeit und subjektiver Workload sind in Tabelle 1 dargestellt. Der durchschnittliche ATI-Score der Stichprobe wich signifikant vom Skalenmittelwert 3,5 ab $(t(15)=3,55, p=.003$, $d=0,89$ ), somit ist die Stichprobe im Mittel als eher technikaffin einzustufen.

Tabelle 1: $\quad$ Deskriptive Statistiken der untersuchten Variablen

Quelle: $\quad$ Eigene Darstellung

\begin{tabular}{lrrrrr}
\hline & \multicolumn{1}{c}{ S } & \multicolumn{1}{c}{ Min } & \multicolumn{1}{c}{ Max } & $n$ \\
\hline Interaktionsbezogene Technikaffinität (ATI) & 4,21 & 0,80 & 2,67 & 5,44 & 16 \\
Interaktionszeit in Sekunden & 269,14 & 77,92 & 148,33 & 413,95 & 14 \\
Subjektiver Workload (NASA-TLX) & 23,47 & 1,94 & 8,33 & 37,50 & 16
\end{tabular}

Für die Beantwortung von Q1 wurden Pearson Produkt-Moment-Korrelationen berechnet. Hierbei zeigte sich bezüglich des Zusammenhangs zwischen ATI und der Interaktionszeit keine signifikante Korrelation: $r=, 05, p=, 858$ (H1 nicht unterstützt). Bezüglich des Zusammenhangs zwischen ATI und dem subjektiven Workload zeigte sich eine signifikante moderate negative Korrelation: $r=-, 46, p=, 036$ (H2 somit unterstützt).

Zur Überprüfung von Q2 wurden zwei multiple lineare Regressionen mit dem ATI-Score sowie der Art des Trainings (Dummy-codiert; kein Training $=0$, angeleitetes Training $=1$ ) als Prädiktoren und der Interaktionszeit bzw. dem subjektiven Workload als Kriterien gerechnet. ${ }^{1}$ Die Ergebnisse sind in Tabelle 2 dargestellt.

Bezüglich der Vorhersage der Interaktionszeit zeigt sich, dass nur das Training, nicht jedoch der ATI-Score einen Effekt zur Vorhersage der Interaktionszeit hat. Bezüglich der Vorhersage

\footnotetext{
${ }^{1}$ Hierbei ist zu beachten, dass für eine multiple Regression mit zwei Prädiktoren eine Stichprobengröße von $n=15$ durchaus als ausreichend beurteilt werden kann, wenn ein entsprechend großer Effekt $\left(\rho^{2}=.70\right)$ in der Population vorliegt (Knofczynski \& Mundfrom, 2007). Da uns der erwartete Populationseffekt nicht bekannt ist, beschränken wir uns bei der Interpretation der Ergebnisse lediglich auf eine deskriptive Beschreibung der Regressionskoeffizienten (d.h. keine Interpretation der Signifikanztestergebnisse).
} 
des subjektiven Workloads fällt im Gegensatz auf, dass der standardisierte Regressionskoeffizient für den ATI-Score wesentlich höher ausfällt als für das Training.

Tabelle 2: $\quad$ Multiple Regressionsmodelle zur Vorhersage der Interaktionszeit und des subjektiven Workloads

Quelle: $\quad$ Eigene Darstellung

\begin{tabular}{|c|c|c|c|c|c|c|c|}
\hline Kriterium & Prädiktor & $B$ & $\beta$ & $p_{\beta}$ & $R_{\text {adj }}^{2}\left(R^{2}\right)$ & $p$ & $n$ \\
\hline \multirow[t]{3}{*}{ Interaktionszeit } & Interaktionsbezogene & & & & \multirow{3}{*}{,45 $(, 54)$} & \multirow{3}{*}{, 014} & \multirow{3}{*}{14} \\
\hline & Technikaffinität (ATI) & 0,33 & ,00 & ,986 & & & \\
\hline & Training & $-111,16$ &,- 73 & ,004 & & & \\
\hline \multirow{3}{*}{$\begin{array}{l}\text { Subjektiver } \\
\text { Workload } \\
\text { (NASA-TLX) }\end{array}$} & Interaktionsbezogene & 161 & & P7? & \multirow{3}{*}{,12 (,24) } & \multirow{3}{*}{, 172 } & \multirow{3}{*}{16} \\
\hline & Technikaffinität (ATI) & $-4,64$ &,- 48 & , U/2 & & & \\
\hline & Training & $-2,31$ &,- 15 &,- 631 & & & \\
\hline
\end{tabular}

\section{DISKUSSION UND SCHLUSSFOLGERUNG}

Auf Basis der bis zum gegenwärtigen Zeitpunkt gesammelten Daten konnte gezeigt werden, dass die interaktionsbezogene Technikaffinität keinen Zusammenhang mit der Interaktionszeit, jedoch einen moderaten negativen Zusammenhang mit dem subjektiven Workload bei einer Aufgabe in einem Industrie 4.0-Kontext aufweist: je höher die Technikaffinität, desto geringer der subjektive Workload bei der Interaktion mit einem 3DDrucker. Dieser Effekt scheint auch weiterhin zu bestehen, wenn man das Durchlaufen einer angeleiteten Trainingseinheit mit in die Analyse einbezieht (allerdings sind die Ergebnisse der multiplen Regressionen aufgrund der geringen Stichprobengröße als erste Hinweise zu verstehen). Bezüglich der Interaktionszeit scheint jedoch das Training (d.h. die Erfahrung) einen wesentlich größeren Einfluss auszuüben als die Technikaffinität.

Die Ergebnisse implizieren, dass sich die interindividuell unterschiedliche Technikaffinität nicht zwangsläufig in der Interaktionszeit widerspiegelt - zumindest bei der gestellten Aufgabe. Dennoch wirkt sich die Technikaffinität auf den subjektiven Workload der Probanden aus. Möglicherweise strengten sich die Probanden mit geringerem ATI-Score mehr an und konnten somit eine ähnlich hohe Performanz zeigen wie die Probanden mit höherem ATI-Score, für die die Aufgabe mit einem geringerem Workload verbunden war. Für die Praxis bedeutet dies, dass die Einbeziehung des ATI-Scores eines Mitarbeiters hilfreich sein kann, um individuelle Trainingsanforderungen abzuleiten. Dies kann möglicherweise Bedienfehlern aufgrund eines zu hohen Workloads vorbeugen und die Mitarbeiter dabei unterstützen, unabhängig von ihrer interaktionsbezogenen Technikaffinität hohe Performanzlevel zu erreichen.

Für stärker untermauerte Befunde bezüglich des Zusammenhangs zwischen der interaktionsbezogenen Technikaffinität und des Interaktionserfolgs ist es wichtig, weitere Performanzmaße (z.B. Fehler) zu untersuchen. Fehlerraten könnten nicht nur weitere Befunde zum Zusammenhang zwischen ATI und Performanz darstellen - gezielte Konfrontation von Probanden mit Fehlern eines technischen Systems könnten auch eine Möglichkeit sein, den 
tatsächlichen Problemlöseprozess von Personen mit hoher und niedrigem ATI-Score zu vergleichen. Zu erwarten wäre hier, dass sich Unterschiede im Interaktionsverhalten vor allem dann zeigen, wenn Probleme auftreten (d.h. Personen mit hohem ATI-Score sollten mit mehr Neugier und Exploration versuchen, die Fehlfunktion zu beheben). Darüber hinaus sollte die Art der Aufgabe experimentell variiert werden, um spezifischere Aussagen für unterschiedliche Kontexte zu ermöglichen. Beispielsweise unterscheiden sich Interaktionszeiten für neuartige Problemlöseaufgaben und gut gelernte Routineaufgaben in Abhängigkeit der Expertise (Goldhammer et al., 2014). Es ist zu erwarten, dass die Ausprägung der interaktionsbezogenen Technikaffinität insbesondere für neuartige Problemlöseaufgaben, die Exploration erfordern, ausschlaggebend ist.

Zusammenfassend konnten die ersten Ergebnisse der vorliegenden Studie zeigen, dass die interaktionsbezogene Technikaffinität große Relevanz für die Vorhersage der individuellen Beanspruchung bei der Interaktion mit technischen Systemen im Kontext von Industrie 4.0 liefert. Dieser Befund ist insbesondere bei der Usability-Evaluation von technischen Systemen zu berücksichtigen.

\section{DANKSAGUNG}

Wir danken unseren studentischen Hilfskräften Jan Gründling, Kim Klüber und Johanna Bake für ihre Unterstützung bei der Studiendurchführung und Videoannotation sowie Michael Bojko für seine aktive Mitarbeit bei der Umsetzung des Versuchskonzeptes.

Die Nutzerstudie wurde im Projekt Factory2Fit durchgeführt und durch H2020, das Rahmenprogramm der EU für Forschung und Innovation [H2020/2014-2020] mit der Vertragsnummer 723277, gefördert. Die Forschungsarbeiten bezüglich des ATI wurden außerdem durch den Europäischen Sozialfond und dem Freistaat Sachsen unter der Antragsnummer 100269974 unterstützt.

\section{LITERATURVERZEICHNIS}

Attig, C., Wessel, D., \& Franke, T. (2017). Assessing personality differences in humantechnology interaction: An overview of key self-report scales to predict successful interaction. In C. Stephanidis (Ed.), HCl International 2017 - Posters' Extended Abstracts, Part I, CCIS 713 (pp. 19-29). Cham, Switzerland: Springer International Publishing AG. doi:10.1007/978-3-319-58750-9_3

Attig, C., Wessel, D., \& Franke, T. (2018, Februar). ATI-eine Skala zur Erfassung von Affinität für Technikinteraktion. Präsentation im Forschungskolloquium des Quality and Usability Lab der Technischen Universität Berlin.

Beier, G. (1999). Kontrollüberzeugungen im Umgang mit Technik. Report Psychologie, 9, 684693. 
Bless, H., Wänke, M., Bohner, G., Fellhauer, R. F., \& Schwarz, N. (1994). Need for cognition: A scale measuring engagement and happiness in cognitive tasks. Zeitschrift für Sozialpsychologie, 25, 147-154.

Cacioppo, J. T., \& Petty, R. E. (1982). The need for cognition. Journal of Personality and Social Psychology, 42, 116-131. doi:10.1037/0022-3514.42.1.116

Cripps, B. (2017). Psychometric testing: Critical perspectives. Chichester, United Kingdom: John Wiley \& Sons.

ELAN (Version 5.2) [Computersoftware]. (04. April 2018). Nijmegen, Niederlande: Max PlanckInstitut für Psycholinguistik. Abgerufen von https://tla.mpi.nl/tools/tla-tools/elan/

Franke, T., Attig, C., \& Wessel, D. (2018). A personal resource for technology interaction: Development and validation of the Affinity for Technology Interaction (ATI) scale. International Journal of Human-Computer Interaction. doi:10.1080/10447318.2018.1456150

Goldhammer, F., Naumann, J., Stelter, A., Tóth, K., Rölke, H., \& Klieme, E. (2014). The time on task effect in reading and problem solving is moderated by task difficulty and skill: Insights from a computer-based large-scale assessment. Journal of Educational Psychology, 106, 608-626. doi:10.1037/a0034716

Hart, S. G., \& Staveland, L. E. (1988). Development of NASA-TLX (Task Load Index): Results of empirical and theoretical research. Advances in Psychology, 52, 139-183. doi:10.1016/S0166-4115(08)62386-9

Hermann, M., Pentek, T., \& Otto, B. (2016). Design Principles for Industrie 4.0 Scenarios. In 2016 49th Hawaii International Conference on System Sciences (pp. 3928-3937). doi:10.1109/HICSS.2016.48

Karrer, K., Glaser, C., Clemens, C., \& Bruder, C. (2009). Technikaffinität erfassen - der Fragebogen TA-EG. In A. Lichtenstein, C. Stößel, \& C. Clemens (Eds.), Der Mensch im Mittelpunkt technischer Systeme. 8. Berliner Werkstatt Mensch-Maschine-Systeme 7. bis 9. Oktober 2009 (pp.196-201). Düsseldorf, Germany: VDI.

Knofczynski, G. T., \& Mundfrom, D. (2008). Sample sizes when using multiple linear regression for prediction. Educational and Psychological Measurement, 68, 431-442. doi:10.1177/0013164407310131

Mahmood, M. A., Burn, J. M., Gemoets, L. A., \& Jacquez, C. (2000). Variables affecting information technology end-user satisfaction: A meta-analysis of the empirical literature. International Journal of Human-Computer Studies, 52, 751-771. doi:10.1006/ijhc.1999.0353

Moosbrugger, H., \& Kelava, A. (2012). Testtheorie und Fragebogenkonstruktion. Berlin, Germany: Springer.

Pinzone, M., Fantini, P., Perini, S., Garavaglia, S., Taisch, M., \& Miragliotta, G. (2017). Jobs and Skills in Industry 4.0: An Exploratory Research. In: H. Lödding, R. Riedel, K. D. Thoben, G. von Cieminski, \& D. Kiritsis (Eds.), Advances in Production Management Systems. The Path to Intelligent, Collaborative and Sustainable Manufacturing. APMS 2017. IFIP Advances in Information and Communication Technology. Cham, Switzerland: Springer. doi:10.1007/978-3-319-66923-6_33

Pocius, K. E. (1991). Personality Factors in Human-Computer Interaction: A Review of the Literature. Computers in Human Behavior, 7, 103-135. doi:10.1016/07475632(91)90002-I 
Richter, T., Naumann, J., \& Horz, H. (2010). A revised version of the computer literacy inventory (INCOBI-R). Zeitschrift für Pädagogische Psychologie, 24, 23-37. doi:10.1024/1010-0652/a000002.

Schmettow M., \& Drees, M. (2014). What drives the geeks? Linking computer enthusiasm to achievement goals. In Proceedings of $\mathrm{HCl}$ 2014, Southport, UK (pp. 234-239). doi:10.14236/ewic/hci2014.29

Windlinger, L., Nenonen, S., \& Airo, K. (2016). Specification and empirical exploration of a usability concept in the workplace. Facilities, 34, 649-661. doi:10.1108/F-01-2015-0003 